\title{
Chitin Nanofibrils in Renewable Materials for Packaging and Personal Care Applications
}

\author{
Maria-Beatrice Coltelli, 2*, Vito Gigante ${ }^{1,2}$, Luca Panariello1, 2, Laura Aliotta1, 2 , \\ Pierfrancesco Morganti $^{3}$, Serena Danti ${ }^{1,2}$, Patrizia Cinelli ${ }^{1,2}$, Andrea Lazzeri ${ }^{1,2}$ \\ ${ }^{1}$ Department of Civil and Industrial Engineering, University of Pisa, Via Diotisalvi 2, Pisa 56122, Italy \\ ${ }^{2}$ National Inter University Consortium of Materials Science and Technology (INSTM), Clo University of Pisa, \\ Via Diotisalvi 2, Pisa 56122, Italy \\ ${ }^{3}$ Skin Pharmacology and Dermatology Unit, Campania University "Luigi Vanvitelli”, Naples, Italy
}

*Corresponding author: Tel: (+39) 0502217856; E-mail: maria.beatrice.coltelli@unipi.it

Received: 15 August 2018, Revised: 08 December 2018 and Accepted: 18 December 2018

DOI: $10.5185 /$ amlett.2019.2250

www.vbripress.com/aml

\begin{abstract}
Chitin nano-fibrils, obtained by waste sea food (for example exoskeletons of crustaceous), are available as water diluted nano-suspensions. Hence, their dispersion at the nanoscale in a molten polyester matrix is considered an issue, because diluted liquids cannot be usually added easily in most common extruders. In the present paper the use of poly(ethylene glycol) (PEG) of different molecular weight was investigated to prepare solid pre-composites useful to disperse chitin nanofibrils in poly(lactic acid) (PLA) by extrusion. The tensile properties of injection moulded specimens were determined and insights were also provided regarding the thermal characteristics of chitin nanofibril-reinforced nanocomposites. This study allowed the identification of a process leading to transparent PLA-based nanocomposites suitable to be exploited in packaging and personal care applications, where the intrinsic anti-microbial and tissue regenerative properties of chitin nanofibrils can be greatly useful. Copyright $\odot$ VBRI Press.
\end{abstract}

Keywords: Chitin, nanofibril, nanocomposite, poly(lactic acid), biopolyesters.

\section{Introduction}

Biodegradable and renewable polyester such as poly(lactic acid) (PLA) can be used for preparing both flexible and rigid products in dependence of the final applications. The use of suitable plasticizers [1-3], nucleating agents [3-4] and processing aids [5] compatible with material renewability and biodegradability is currently the objective of many researches. Indeed, the preparation of nanocomposites [6-9], especially with natural nanofillers [10-11], aims at exploring the potentialities of nanotechnology to overcome application-related issues. The main goal is preparing materials which are more benign towards environment and health, and use them in packaging or personal care applications to replace petro-sourced counterparts [12-15]. Several natural biopolymers showed interesting biocidal properties suitable for packaging applications: chitosan, obtained by the deacetylation of chitin, gelatin, pectin, cationic dextran and cationic cellulose [16].

Chitin represents the second most abundant biomass resource on earth with global reserves of 100 billion tons [15]. It is present as main component of the exoskeleton of crustaceous and insects and can be obtained by sea food waste that is globally produced in large amount. Chitin nano-fibrils (CNs) are obtained in aqueous suspension from chitin and have nanometric dimensions.
It is demonstrated that they possess anti-microbial activity and favor tissue regeneration [17]. CNs can be easily dispersed in water-soluble polymers, such as chitosan in an acidic environment using water as a solvent [18]. The removal of water allows casting films [19] or coagulation fibers [20] to be obtained. Differently, the dispersion in non-water-soluble polymers, and indeed, sensitive to moisture during processing, such as biodegradable polyesters, is much more difficult. On the other hand, these polymers are processable by injection molding, thermoforming, flat die extrusion and extrusion, and therefore for a quick transfer into application in different sectors it is necessary for the nanofibrils to be successfully dispersed inside polymer matrices in the molten state. In particular, the extrusion of granules in which the CNs are dispersed as large microsized aggregates are characterized by high fragility and low transparency, features that prevent both the processing and the use.

The obtainment of the nano-dispersion, being necessary to have industrially biodegradable plastic objects containing CNs, also allows CNS to be exploited as reinforcement agents for the modulation of the mechanical properties [21], nucleating agents [22], antimicrobial agents as a function of the degree of acetylation and $\mathrm{pH}$ [23], or as carrier of substances, such as dyes [24], antioxidants and active substances for potential applications in the intelligent packaging sector 
or personal care/cosmetic sector [14-15]. Zhang et al. [25] have acetylated chitin to improve dispersion in poly (lactic acid) (PLA), but the tensile properties of composites prepared by solvent casting (not in the molten state) showed a low value of elongation at break, according to a suboptimal dispersion of CNs.

Rizvi et al. [23], evaluated the effects of different amounts of chitin nano-fibrils in melt-blended PLA/chitin and PLA/CN composites. Given the hydrophobic nature of PLA, which implies poor adhesion at the interface with natural reinforcements that are hydrophilic, a series of nano-composites has been prepared with the addition of a compatibilizing agent. Therefore, PLA nano-composites were prepared on which maleic anhydride (MA) was grafted to PLA at about $2 \%$ by weight producing a modified polymer (PLA-g-MA). Tensile tests showed an increase in Young's modulus in nano-composites compared to pure PLA. In particular, it has been observed that the Young's modulus underwent an increase up to $5 \%$ chitin content, while higher quantities did not produce further reinforcement effect. As regards the tensile strength, a decrease by increasing the nano-chitin content was observed and, in any case, always lower values than pure PLA. Such a decreasing trend was attributed to the hydrolysis of PLA during preparation, since aqueous solutions of CNs were used to avoid phenomena of agglomeration, and therefore, as the amount of reinforcement used increased, the introduced moisture increased accordingly, leading to the hydrolysis of the PLA. This paper showed the good potentialities of CNs to improve mechanical performance but at the same time it highlighted the difficulty of dispersing them at the nano-metric level and homogeneously in the PLA. Guan and Naguib [21] studied PLA/CN nano-composites using MA as a compatibilizing agent and N, Ndimethylacetamide (DMAc) as a dispersing agent, considered necessary to improve the dispersion of nanofibrils in composite. The CNs were re-dispersed in DMAc by mechanical stirring to enable a nanostructured suspension. Moreover, to favor the fibermatrix adhesion, PLA was prepared with grafted anhydride groups (PLA-g-MA) using $2 \mathrm{wt} \%$ of MA and $0.5 \mathrm{wt} \%$ of peroxide as a radical initiator. Tensile tests have demonstrated that the presence of both DMAc and PLA-g-MA, without CNs, led to a decrease of the Young's modulus and of the tensile strength; DMAc caused the reduction of the mechanical properties due to, according to the authors, the degradation generated in the PLA through chain scission during the extrusion. In the specimens that presented both the $\mathrm{CNs}$ as nanostructured suspension and compatibilization agents, an increase in the modulus of elasticity and tensile strength could be observed, so that it was concluded that the presence of nano-fillers counterbalanced the degradation effect. An increase of Young's Modulus and tensile strength with reduction of the deformation at break was detected in these nano-composites. It is also important to note that, as the amount of nano-filler increased, two contrasting effects occurred: (i) the reinforcing effect of chitin, which increased stiffness and strength at the expense of ductility; and (ii) a negative impact on mechanical properties attributable to the presence of DMAc which caused the degradation of the matrix.

This article underpinned the need to use a dispersing agent, looking for one that does not negatively impacts the mechanical performances of PLA. The use of known plasticizers of PLA in PLA-based nanocomposites has been studied by several researchers and poly(ethylene glycol) (PEG) was successfully used to disperse CNs $[12,26]$ in PLA. Successively, other researchers came to similar conclusions [27-30].

Herrera et al. [27-29] developed citrate in water/alcohol suspensions and investigated the properties of composites containing $1 \%$ of CNs. They reported that this amount was sufficient to modify some key properties, such as anti-fungal activity, antistatic behavior and puncture strength, thanks to the very high surface to volume ratio of nanofibrils. More recently $\mathrm{Li}$ et al. [30], aiming at obtaining rigid nanocomposites, used high molecular weight poly(ethylene oxide) (PEO) or PEG and investigated the flexural and impact properties of composites with $\mathrm{NC}$ in the range $10 \%$ $40 \%$. The use of PEG and PEO was considered less effective than other methods, without any plasticizing agent, due to the decrease of the elastic modulus attributable to plasticization. However, this methodology based on PEG can be advantageous in the case of study of flexible materials, to be applied in plastic films, with low content of CNs.

Moreover, more research is required to identify specific procedures for preparing these nanocomposites, considering also extrusion protocols and processing parameters, that highly affect the final properties of the composite.

In this frame, in the present paper the use of PEGs of different molecular weight is proposed to disperse CNs in PLA thanks to the preparation of pre-composites. The pre-composites are then dispersed in PLA during extrusion to obtain plasticized PLA containing $2 \%$ by weight of NC. The tensile properties of injection molded specimens are discussed, and insights were also provided regarding the thermal characteristics of $\mathrm{CN}$ - reinforced nanocomposites.

\section{Experimental}

\section{Materials / chemicals details}

CN water suspension was produced by MAVI SUD through its patented process [31] and concentrated up to $20 \%$ by weight. PEG having a molecular weight of 400 (P4), liquid, and PEG having a molecular weight of 4000 (P40), 6000 (P60) and 8000 (P80), solid, purchased from Aldrich, were used without any further purification.

PLA Ingeo TM 2003D, Extrusion Grade with density of $1,24 \mathrm{~g} / \mathrm{cm}^{3}$, a melt index of $6 \mathrm{~g} / 10 \mathrm{~min}$ at $210{ }^{\circ} \mathrm{C}$ and $2,16 \mathrm{Kg}$, produced by NatureWorks LLC. It has a molecular weight of $170000 \mathrm{~g} / \mathrm{mol}$ and contains up to $4.1 \%$ isomeric D units. It was dried in ventilated oven at $60{ }^{\circ} \mathrm{C}$ for 16 hours before extrusion trials. 


\section{Material synthesis / reactions}

PG4 (or PG80) were added to concentrated chitin nanofibrils suspension and stirred for two hours at room temperature. The amount was calculated considering that in the final pre-composites the weight ratio of PEG and $\mathrm{NC}$ was 1:1. The obtained semiliquid emulsion was dried in a ventilated oven at $50{ }^{\circ} \mathrm{C}$ up to constant weight to obtain a solid when P80 was employed (P80S, P40S and P60S) and a sample with liquid highly viscous consistency when P4 was used (P4S).

The extrusion of the PLA 2003D (Ingeo TM Nature Works) in the presence of P80S or P4S and PEG was carried out, after drying the material, using a TwinLab II Haake $^{\mathrm{TM}}$ Rheomex CTW 5 laboratory screw extruder. The extrusion was carried out at $180^{\circ} \mathrm{C}$ and $90 \mathrm{rpm}$ for one minute. After extrusion, the molten material was transferred through a preheated cylinder into the Haake TM MiniJet II mini injection molding machine to obtain Haake type III test specimens for tensile testing. The injection molding was carried out at $180{ }^{\circ} \mathrm{C}$, 650 bar, holding time of 15 seconds, mold temperature of $35^{\circ} \mathrm{C}$.

The extrusion and injection molding were carried out onto: pure PLA (PLA); PLA with 2\% wt. of dried CNs (PLA+2CN*); PLA plasticized with $\mathrm{P} 4$ at $10 \%$ by weight (PLA +P4-10); PLA containing $10 \%$ wt. of P4 and $2 \%$ wt. of $\mathrm{CNs}$ added by P4S (PLA+P4-10+2CN); PLA containing $10 \%$ wt. of P80 and $2 \%$ wt. CNs added by P80S (PLA+P80-10+2CN);

\section{Characterizations / device fabrications /response measurements}

Tensile tests (UNI EN ISO527) were carried out using a universal INSTRON 5500R test machine with a $1 \mathrm{kN}$ load cell at a speed of $10 \mathrm{~mm} / \mathrm{min}$.

Differential scanning calorimetry analyzes (DSC) were performed on material sampled from injection molded specimens using a TA Q200 instrument with nitrogen as carrier gas and indium as a calibration standard. The samples were heated from $-100{ }^{\circ} \mathrm{C}$ to $200{ }^{\circ} \mathrm{C}$ at $10{ }^{\circ} \mathrm{C} / \mathrm{min}$ to clean the thermal history of the sample and cooled from $200{ }^{\circ} \mathrm{C}$ to $-100{ }^{\circ} \mathrm{C}$ at $20{ }^{\circ} \mathrm{C} / \mathrm{min}$. The second heating was carried out by heating analogously from $-100{ }^{\circ} \mathrm{C}$ to $200{ }^{\circ} \mathrm{C}$ at $10{ }^{\circ} \mathrm{C} / \mathrm{min}$.

The morphology of master batches and composites was studied by scanning electron microscopy (SEM) using a JEOL JSM-5600LV instrument and analyzing cryo-fractured surfaces, previously subjected to sputtering with gold.

\section{Results and discussion}

If a suspension of CNs is deposited in a Petri dish a film is formed after drying and its structure consists of strongly aggregated chitin nanofibrils (Fig. 1). In Fig. 1a it is possible to observe how the drying of nanosized chitin has produced large flakes in which the fibers are aggregated to form a compact structure similar to that of the paper. Here, the nano-fibrous consistency is observable only at the edges of the flakes. (a)

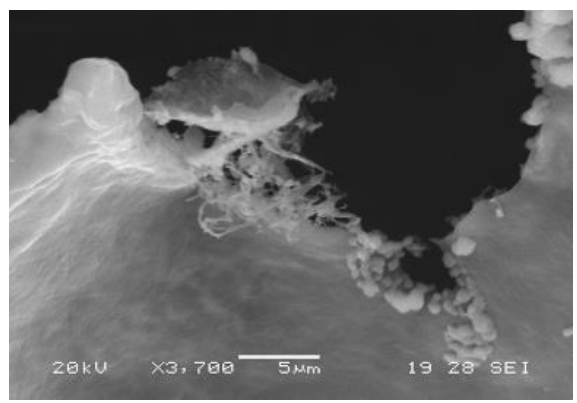

(b)

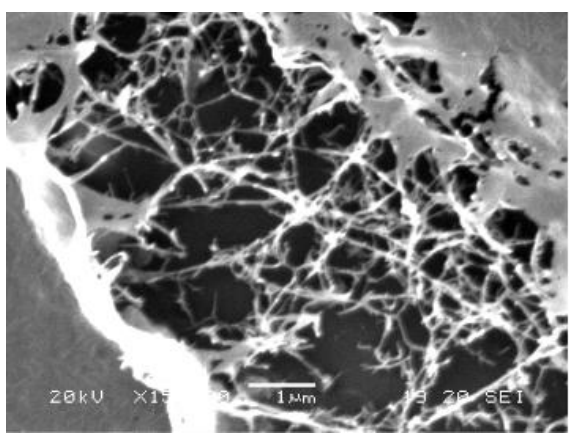

Fig. 1. SEM micrographs related to films obtained by drying CNs from water suspensions: (a) $3700 \times$ magnification; (b) $15,000 \times$ magnification.

P80 was analyzed by SEM (Fig. 2) and it was possible to observe the maintenance of the nano-fibrous structure (Fig. 2b). Reasonably the use of the plasticizer therefore allows the nanofibrils to be kept well separated, thus avoiding their aggregation.

(a)

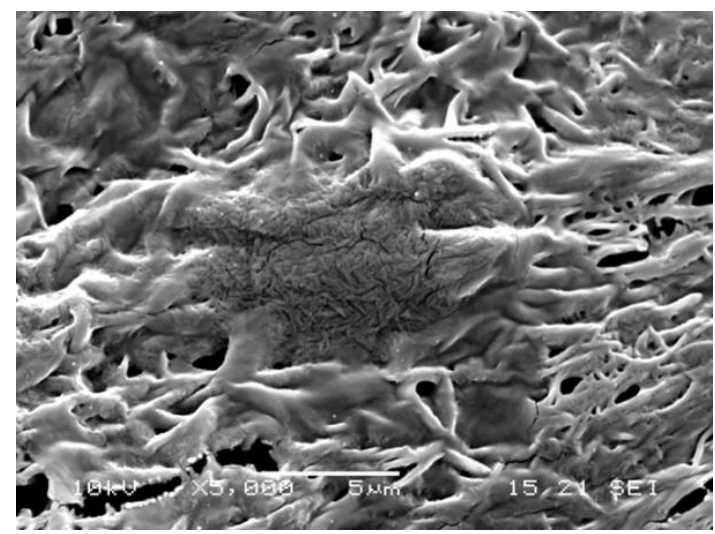

(b)

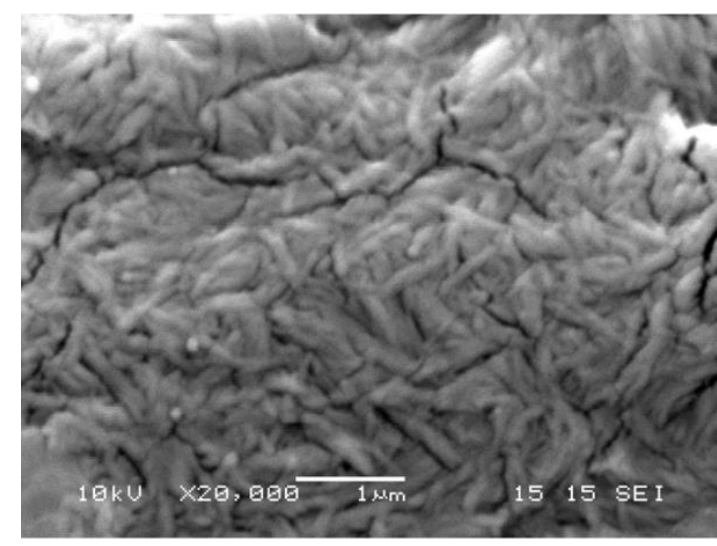

Fig. 2. SEM micrographs related to the pre-dispersed solid prepared by using P80 poly(ethylene glycol) (P80S): (a) 5000x magnification; (b) $20,000 \times$ magnification. 
It was not possible to make a similar analysis of the $\mathrm{P} 4 \mathrm{~S}$, which gave rise to a deformable viscous paste. Anyway, P40S and P60S showed a morphology quite similar to P80S (Fig. 3).

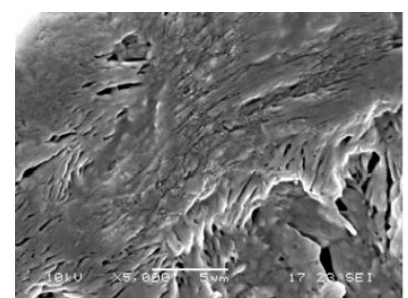

(a)

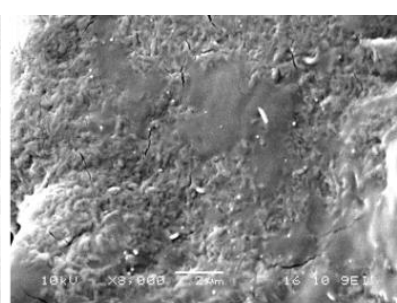

(b)
Fig. 3. SEM micrographs related to the pre-dispersed solid prepared by using (a) P40 poly(ethylene glycol) (P40S) and (b) P60 (P60S)

P4S and P80S were added to PLA in a laboratory scale twin-screw extruder and nano-composites were thus prepared. In fact, transparent extruded strands and injection molded specimens were obtained from these trials (Fig. 4b) and the absence of micrometric agglomerates was also confirmed by SEM analysis. Interestingly, it can be noticed the presence of almost spherical dispersed domains attributable to the presence of PEG partially in separated domains, due to the incomplete miscibility of this plasticizer with PLA [32]. On the contrary, by adding dried CNs to PLA, opaque micro-composites were obtained which were characterized by the presence of nanofibrils agglomerates (Fig. 4a).

It is thus evident that, by adding none, presence of agglomerates is revealed.


Fig. 4. SEM micrographs and picture of specimens related (a) microcomposite obtained by dried chitin nanofibrils in PLA at $2 \%$ wt. $(\mathrm{PLA}+2 \mathrm{CN})$; (b) nano-composites obtained by using $\mathrm{P} 4$ and the $\mathrm{P} 4$ precomposite (PLA+P4-10+2CN).
The tensile properties were measured, and it was found that the dispersion in PLA of dried nanofibrils, hence aggregated (Table 1), resulted in a decrease of elastic modulus, stress at break and elongation at break due to the increased brittleness. On the contrary, the addition of CNs using the methodology based on the use of P4 and P80 allowed the obtainment of materials with an elongation at break quite similar to the plasticized sample without nanofibrils. Although the CNs did not enhance the elastic modulus and the stress at break, the maintenance of a high elongation at break indicated that a nanoscaled dispersion was achieved, in agreement with morphologic and optical evaluations. Thanks to this good dispersion, high interfacial surface and low dimension, the nanofibrils do not contribute to increase the brittleness of the material, as instead has generally been observed for many microfillers [33].

Table 1. Tensile properties of PLA based CN- containing composites.

\begin{tabular}{l|c|c|c}
\hline & $\begin{array}{c}\mathbf{E} \\
(\mathbf{G P a})\end{array}$ & $\begin{array}{c}\boldsymbol{\sigma}_{\mathbf{b}} \\
(\mathbf{M P a})\end{array}$ & $\begin{array}{c}\boldsymbol{\varepsilon}_{\mathbf{b}} \\
(\boldsymbol{\%})\end{array}$ \\
\hline PLA & $3.5 \pm 0.1$ & $57 \pm 1$ & $4.1 \pm 0.5$ \\
\hline PLA+2CN* & $2.9 \pm 0.1$ & $58 \pm 2$ & $2 ., 3 \pm 0.4$ \\
\hline PLA +P4-10 & $2.3 \pm 0.3$ & $33 \pm 2$ & $180.0 \pm 10.0$ \\
\hline PLA+P4-10+2CN & $1.8 \pm 0.3$ & $32 \pm 2$ & $160.0 \pm 10.0$ \\
\hline PLA+P80-10+2CN & $2.5 \pm 0.1$ & $34 \pm 2$ & $160.0 \pm 10.0$ \\
\hline
\end{tabular}

The composites were characterized by DSC tests. The second heating thermograms were analyzed (Table 2 and Fig. 5) and it was found that the thermal properties were not significantly changed by adding chitin nanofibrils, although the decrease of the cold crystallization temperature and the decrease of its enthalpy indicated a slight influence of the $\mathrm{CNs}$ on crystals formation. As the $\Delta \mathrm{Hm}$ does not change significantly by adding CNs to plasticized PLA, the crystallinity content is not modified too. Despite of the high surface to volume ratio of nanofibrils and its good dispersion, the glass transition temperature was not increased with respect to the plasticized PLA, as often observed in nanocomposites [34, 35], but slightly decreased. This can indicate only reduced interactions of nanofibrils surface with the polymer, probably thanks to the presence of PEG on nanofibrils surface. It is thus evident a good synergy with PEG, as CNs do not seem to reduce the plasticizing effect of this additive.

Table 2. DSC results related to chitin nanofibrils PLA based nanococmposites.

\begin{tabular}{l|c|c|c|c|c}
\hline & $\begin{array}{c}\mathbf{T}_{\mathbf{g}} \\
\left({ }^{\circ} \mathbf{C}\right)\end{array}$ & $\begin{array}{c}\mathbf{T}_{\text {cc }} \\
\left({ }^{\circ} \mathbf{C}\right)\end{array}$ & $\begin{array}{c}\Delta \mathbf{H}_{\mathrm{cc}} \\
(\mathbf{J} / \mathbf{g})\end{array}$ & $\begin{array}{c}\mathbf{T}_{\mathbf{m}} \\
\left({ }^{\circ} \mathbf{C}\right)\end{array}$ & $\begin{array}{c}\Delta \mathbf{H}_{\mathbf{m}} \\
(\mathbf{J} / \mathbf{g})\end{array}$ \\
\hline PLA & 59.1 & 109.4 & 23.8 & $148.3 / 157,4$ & 2.6 \\
\hline PLA+2CN* & 55.2 & 108.8 & 23.3 & $147.9 / 155,8$ & 1.2 \\
\hline PLA + P4-10 & 36.1 & 83.8 & 21.3 & $153, .7$ & 11.3 \\
\hline PLA+P4-10+2CN & 32.7 & 79.8 & 17.1 & 152.1 & 12.1 \\
\hline PLA+P80-10+2CN & 31.4 & 80.5 & 17.5 & 154.2 & 11.8 \\
\hline
\end{tabular}

$\mathbf{T}_{\mathrm{g}}=$ glass transition temperature; Tcc $=$ crystallization during heating peak temperature; $\Delta \mathrm{Hcc}=$ enthalpy of crystallization during heating; $\mathrm{Tm}=$ melting peak temperature; $\Delta \mathrm{Hm}=$ integral of the melting peak$\Delta \mathrm{Hcc}$ 


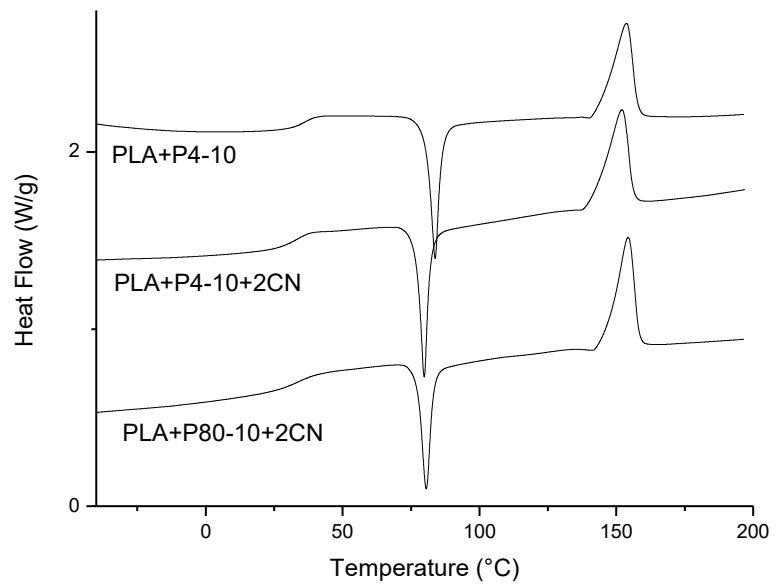

Fig. 5. Second heating thermograms of PEG400 plasticized PLA, the same with $2 \%$ by weight of $\mathrm{CNs}$ and the same plasticized with PEG8000 and with $2 \%$ of CNs. The thermograms were vertically translated to allow a better comparison

In general, mechanical and thermal characterizations showed that CNs are nano-dispersed in PLA based plasticized films without significantly altering their flexibility and crystallization behavior.

CNs were thus found as dispersible in PLA and this opened the possibility to add them to biopolyester composites to exploit the intrinsic anti-microbial and tissue regenerative properties of the nano-fibrils allowing a wide spectrum of applications of innovative, nano-structured, renewable and biodegradable materials. These can be applied, through different processing options, in packaging, personal care and biomedical field (Fig. 6).
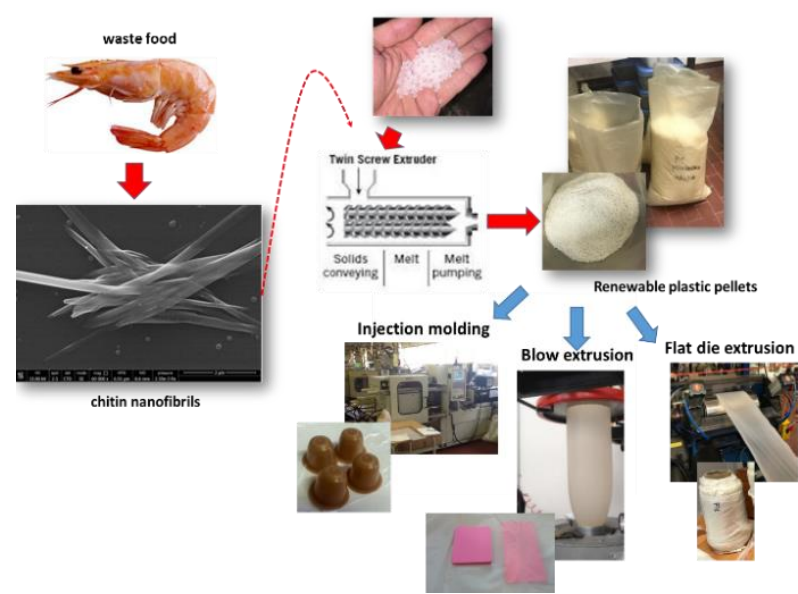

Fig. 6. Platform of processing methodologies and applications considered in past and ongoing European projects about biopolyesters based nano-composites containing CNs.

\section{Conclusion}

In the present paper, the use PEG of different molecular weight (8000 and 400) was successfully used to disperse chitin nanofibrils in PLA thanks to the preliminary preparation of CN/PEG pre-composites. The tensile properties results, related to the transparent injection molded specimens obtained by adding the pre-composite in molten PLA during the extrusion, showed that the mechanical properties of plasticized PLA were not affected by the presence of CNs. The thermal characteristics of the nanocomposites was investigated by DSC measurements and it was found that the presence of CNs, despite of a slight alteration of crystallization during heating temperature and related heat, did not influence significantly thermal properties. These findings can pave the way for developing biobased and biodegradable nanocomposites with improved functionalities containing these anti-microbial and tissue regenerating nanofibrils, suitable to be used in a large range of applications (packaging, personal care and biomedicine) where it is beneficial, considering health and environment concerns, replacing petrochemical plastics.

\section{Acknowledgements}

This work was supported by European Union that financed the projects Nano-Chitopack (Sustainable technologies for the production of biodegradable materials based on natural chitin-nanofibrils derived by waste of fish industry, to produce food grade packaging, G.A. $\mathrm{n}^{\circ}$. 315233) and POLYBIOSKIN (High performance functional bio-based polymers for skin-contact products in biomedical, cosmetic and sanitary industry, G.A. $\mathrm{n}^{\circ}$. 745839). The latter is still ongoing. Microtec (Italy) and Aroma (Italy) companies are thanked for collaborating in prototype developments of films and coffee capsules respectively in the frame of Nano-chitopack project.

\section{Author's contributions}

Conceived the plan: mbc, pc, al; Performed the experiments: vg, lp; Data analysis: la, mbc; Wrote the paper: $\mathrm{mbc}, \mathrm{pm}$, sd. Authors have no competing financial interests.

\section{References}

1. Coltelli, M. B.; Della, Maggiore, I.; Bertoldo, M.; Signori, F.; Bronco, S.; Ciardelli, F.; J. Appl. Polym. Sci., 2008, 110, 1250.

2. Mallegni, N.; Phuong, T. V.; Coltelli, M. B.; Cinelli, P.; Lazzeri, A.; Materials, 2018, 11, 148.

3. Fehri, M. K.; Mugoni, C.; Cinelli, P.; Anguillesi, I.; Coltelli, M. B.; Fiori, S.; Montorsi, M.; Lazzeri, A.; eXPRESS Polym. Lett. 2016, 10, 274.

4. Aliotta, L.; Cinelli, P.; Coltelli, M. B.; Righetti, M. C.; Gazzano, M.; Lazzeri, A.; Eur. Polym. J. 2017, 93822.

5. Al-Itry, R.; Lamnawar, K.; Maazouz, A.; Polym. Degrad. Stab., 2012, 97, 1898.

6. Coltelli, M. B., Coiai, S., Bronco, S.; Passaglia, E.; Nanocomposites Based on Phyllosilicates: from petrochemical to renewable thermoplastic matrices, In Advanced nanomaterials, Geckeler, K. E.; Nishide, H.; A.P.F. (Eds.); Wiley: USA, WileyVCh, Wieinheim, 2010, pp. 403.

7. Raques, J. M.; Habibi, Y.; Murariu, M.; Dubois, P.; Prog. Polym. Sci., 2013, 38, 1504.

8. Castiello, S., Coltelli, M. B.; Conzatti, L.; Bronco, S.; J. Appl. Polym. Sci. 2012.

9. Scatto, M.; Salmini, E.; Castiello, S.; Coltelli, M.B.; Conzatti, L.; Stagnaro, P.; Andreotti, L.; Bronco, S.; J. Appl. Polym. Sci., 2012

10. Fortunati, E.; Luzi, F.; Puglia, D.; Petrucci, R.; Kenny, J.M.; Torre, L.; Ind. Crops Prod., 2015, 67, 439.

11. Scaffaro, R.; Botta, L.; Lopresti, F.; Maio, A.; Sutera, F.; Cellulose 2017, 24, 447.

12. Cinelli, P.; Coltelli, M.B.; Mallegni, N.; Morganti, P; Lazzeri, A.; Chem. Engin. Trans., 2017, 60, 1

13. Morganti, P., Coltelli, M.B., Danti, S.; Glob. J. nanomed. 2018, 3,1 .

14. Morganti, P.; Coltelli, M.B.; Danti, S.; Bugnicourt, E.; Glob. Res. J. Pharm. Pharmacol., 2017, 2, 7 .

15. Morganti, P.; Fusco, A.; Paoletti, I., Perfetto, B.; Del Ciotto, P.; Palombo, M.; Chianese, A.; Baroni, A.; Donnarumma, G.; Materials, 2017, 10, 843. 
16. Kim, S.K. (Ed.); Chitin, Chitosan, Oligosaccharides and Their Derivatives; CRC Press: USA, 2011

17. Chauhan N.P.S. (Ed.); Biocidal polymers; Smither Rapra: UK, 2016

18. Mikesová, J.; Haseka, J.; Tishchenko, G.; Morganti, P.; Carbohydr. Polym., 2014, 112, 753 .

19. Morganti, P.; Tischenko, G.; Palombo, M.; Kelnar, I.; Brozova, L.; Spirkova, M., Pavlova, E.; Koera, L.; Carezzi, F.; Chitin nanofibrils in biomimetic products: nanoparticles and nanocompositechitosan films iin health care, In Marine Biomaterials: Characterization, Isolation and Applications, Kim, S.K.; A.P.F. (Eds.); CRC Press: USA, 2013, pp. 681.

20. Yudin, V.E.; Dobrovolskaya, I. P.; Neelov, I. M.; Dresvyanina, E.N.; Popryadukhin, P.V.; Ivankova, E.M.; Elokhovski, V.Y.; Kasatkin, I.A.; Okrugin, B.M.; Morganti, P.; Carbohydr. Polym. 2014, 108, 176.

21. Guan, Q.; Naguib, H.E.; J. Polym Environ, 2014, 22, 119.

22. Rizvi, R.; Cochrane, B.; Naguib, H.; Lee, P.C.; J. Cell. Plast., 2011, 47, 283.

23. Mincea, M.; Negrulescu, A.; Ostafe, V.; Rev. Adv. Mater. Sci. 2012, 30, 225 .

24. Majeti, N. V.; Kumar, R.; React. Funct. Polym., 2000, 46, 1.

25. Zhang, Q.; Wei, S.; Huang, J.; Feng, J.; Chang, P. R.; J. Appl. Polym. Sci. 2014.

26. Coltelli, M. B.; Cinelli, P.; Anguillesi, I.; Salvadori, S.; Lazzeri, A.; Structure and properties of extruded composites based on biopolyesters and nano-chitin, Session M: Functional Textiles-from xresearch and development to innovations and industrial uptake, Symposium E-MRS Fall meeting, Warsaw, Poland, Book of Abstracts, 192, 2014,

27. Herrera, N.; Salaberria, A. M.; Mathew, A. P., Oksman, K.; Composites: Part A, 2016, 83, 89 .

28. Herrera, N.; Roch H.; Salaberria, AM.; Pino-Orellana, MA.; Labidi, J.; Fernandes, S.C.M.; Radic, D.; Leiva, A.; Oksman, K.; Mater. Des., 2016, $92,846$.

29. Herrera, N.; Singh, A.A.; Salaberria, A.M.; Labidi, J.; Mathew, A.P.; Oksman, K.; Polymers, 2017, 9, 406.

30. Li, J.; Gao, Y.; Zhao, J.; Sun, J.; Li, D.; Cellulose 2017, 24, 1705.

31. Muzzarelli, C.; Morganti, P.; U.S. Patent 8383157 B2, 2013.

32. Hu, Y.; Hu, Y. S.; Topolkaraev, V.; Hiltner, A.; Baer, E.; Polymer, 2003, 44, 5681.

33. Zhang, M. Q.; Rong, M. Z.; Ruan, W. H.; Nanoparticles/Polymer composites: fabrication and mechanical properties, In Nano-and Micro-Mechanics of polymer blends and composites; KargerKocsis, J.; Fakirov, S., (Eds.); Hanser: Germany, 2009, pp. 93.

34. Ahn, S. I.; OhK, C. W.; Kim, J. H.; Zin, W. C.; J. Polym. Sci., Part B: Polym. Phys., 2009, 47, 2281

35. Xiong, M.; Gu, G., You, B.; Wu, L.; J. Appl. Polym. Sci., 2003, 90, 1923. 